\title{
PERSPECTIVE VERGENCE: OCULOMOTOR RESPONSES TO LINE DRAWINGS
}

\author{
J. T. ENRIGHT \\ Neurobiology Unit, A-002, Scripps Institution of Oceanography, La Jolla, CA 92093, U.S.A. and \\ Abteilung Medizinische Optik, Augenklinik der Universität Hamburg, D-2000 Hamburg 20, F.R.G.
}

(Received 19 September 1986; in final revised form 6 April 1987)

\begin{abstract}
When a perspective drawing is viewed monocularly, changes in fixation point are accompanied by changes in steady-state vergence; their direction is usually appropriate for the distance relationships implied in the illustration. The absolute magnitude of these responses varies appreciably among subjects; it can be consistently enhanced or reduced by modest changes in the drawing. Similar configurations of stimuli from three-dimensional objects would presumably also contribute to normal vergence movements during binocular viewing; it appears that their importance would increase with target distance. Corresponding changes in pupil diameter, as expected for the "near reflex", were not observed with perspective stimuli. Consistent, directionally appropriate vergence changes, paralleling perception, were also made by most subjects during monocular viewing of a Necker cube, but there, exceptionally large pupillary responses arose.
\end{abstract}

Eye-movements Vergence Proximal vergence Perspective vergence Pupil Near triad Necker cube

\section{INTRODUCTION}

When we shift our gaze among targets of interest which are at different distances away, convergence and divergence of the eyes are essential to prevent diplopia. The extent to which the images from the two eyes are initially out of register with each other is defined as the disparity of a target, and it represents a strong stimulus for evoking vergence movement-and the essential one for achieving binocular fusion. A second important stimulus for vergence changes is provided by image misfocus; and since perception of image blurring is a central element in the feedback loop controlling accommodation of the lens, the vergence response to such stimuli is known as accommodation vergence. This response is easily measured during monocular viewing, and its magnitude is quite large-typically half or more of the movement required to achieve proper realignment of the two eyes. Nevertheless, accommodation vergence is neither necessary for achieving binocular fusion: disparity stimuli can do the job alone; nor sufficient: only disparity can provide the brain with precise feedback about image superposition. Accommodation vergence is thus in some senses a backup system: a non-essential but powerful supplementary mechanism for moving the eyes in the proper direction toward fusion and a "cyclopean" view of the world.

This article describes evidence for another sort of supplementary mechanism which can produce vergence movements in the appropriate direction: an eye-movement response to perspective stimuli, that is, to those configurations of elements which serve, in a perspective illustration, to indicate that one object is at a greater or lesser distance from the observer than another. Vergence changes which are evoked by this particular sort of stimulus have apparently not been previously reported, but the responses may well represent a subcategory of what is known as "proximal vergence", said to be due to "knowledge of nearness" (Maddox, 1893; Hokoda and Ciuffreda, 1983).

Like accommodation vergence, the oculomotor response to the depth relationships implied by illustrations can be most easily and unequivocally demonstrated under open-loop conditions: disparity stimuli are eliminated by obstructing vision in one eye. Accommodation stimuli are not eliminated in this experimental design, but, since the elements of a drawing are in a plane at a single distance, the stimuli for accommodation are held constant; hence, any 
observed vergence changes must be due to the arrangement of lines within the drawing rather than to differences in image blur.

\section{METHODS}

The video system used to evaluate eye position has been previously described (Enright, 1984); in brief, the position of each eye is monitored from somewhat below the line of sight, using two video cameras with zoom lenses; the two pictures are combined by means of an image splitter, and recorded for subsequent measurement. The system now also includes a field counter which superimposes its numbers during recording $(60 / \mathrm{sec})$, so that each picture is individually identifiable. Replicate "blind" readings indicate that at the magnification used (about 7-fold on the monitor), typical single-measurement precision (reproducibility) corresponds to a standard deviation of about 6 arcmin of ocular rotation; since a single estimate of vergence state involves summing separate measurements of the position of each eye, and since change in vergence is a difference between two estimates of vergence state, the single-measurement precision, for estimates of change in vergence, is about 12 arcmin. Averaging over ten replicates of a given task, as done here, reduces the impact of random measurement error to about 4 arcmin (i.e. $12 / \mathrm{SQRT}[10]$ ), so in a majority of the data (typical standard error of 6 or 7 arcmin), the uncertainty of the estimates includes appreciable trial-to-trial variability in eye position, which could not be eliminated by a measuring system with greater precision. Evaluations of vergence based on monitoring only the frontal view of each eye are subject to potentially serious systematic errors due to lateral translation of the globe (Enright, 1984a). Comparisons between measured vergence changes and geometric expectations indicate, however, that when the changes in vergence are small, and when averages are calculated over several replications of a given eye movement, this problem is sufficiently small that it would not seriously contaminate results of the sort reported here (Enright, 1986; see, also, Discussion). Singlemeasurement precision in the evaluation of pupil diameter (standard deviation of replicate "blind" readings) is about $50 \mu \mathrm{m}$ (about $1 / 3 \mathrm{~mm}$ on the monitor), so random measurement error, in calculation of mean changes in pupil di- ameter, based on $N$ of 10 , could account for an uncertainty in those estimates of about $20 \mu \mathrm{m}$.

The pictures and objects used as targets were mounted about $30 \mathrm{~cm}$ in front of the eyes along the midline, and were continuously visible to the subjects. Each of the illustrations was drawn on a separate piece of white cardboard, about $5 \mathrm{~cm}$ high and $8 \mathrm{~cm}$ wide. All drawings were viewed monocularly; an opaque black screen was placed about $5 \mathrm{~cm}$ in front of the subject's non-dominant eye, at a vertical level which completely obstructed a view of the target, without interfering with the camera's view of that eye. A custom-fitted bite board and forehead rest were used to maintain head stability.

During a typical recording session, the subject alternated fixation, in response to verbal commands given at intervals of $2-4 \mathrm{sec}$, between the "front" and the "back" corners of a rectangular box, either in one of the drawings [Fig. I(A-E)], or on the corresponding corners of a real box. Those real boxes were constructed from thin white cardboard, and appropriately marked with black lines so as to look like the illustration of either Fig. 1(A) or Fig. 1(D); one of those boxes [matching Fig. 1(A)] was $28 \mathrm{~mm}$ across the interior diagonal of the opening, and the other [matching Fig. 1(D)] was $56 \mathrm{~mm}$. They were mounted at about the same distance $(30 \mathrm{~cm})$ and elevation as the illustrations, and tilted and rotated so as to provide a satisfactory (monocular) match to the corresponding drawing. The two fixation points in the drawings as well as on the cardboard boxes required that the dominant eye (the viewing eye in all monocular tests) move diagonally, upward and slightly to the right, and then back again, with about $2.5^{\circ}$ vertical movement and about $0.5^{\circ}$ horizontal movement. Calibration measurements, for converting eye movements from $\mathrm{mm}$ to degrees, were made during binocular viewing of fixation markers which were about $6^{\circ}$ apart in the horizontal plane, at $30 \mathrm{~cm}$ distance.

In a typical test session, there were 10 changes of fixation in each direction, between the front and the back corners of the box. During the recording, the subjects indicated which corner was being fixated by depressing a three-position switch, which was connected to two different tone generators. For the perspective drawings [Fig. 1(A-E)], the timing of the change in fixation was also evident by the near-vertical saccade; when viewing the Necker-cube drawing [Fig. 1(F)], the tone served to define perception of the orientation, that is, whether the fixated 

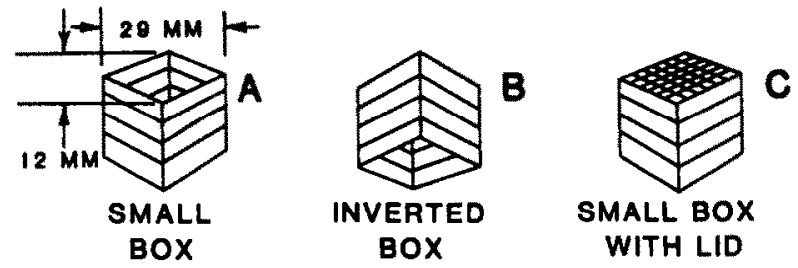

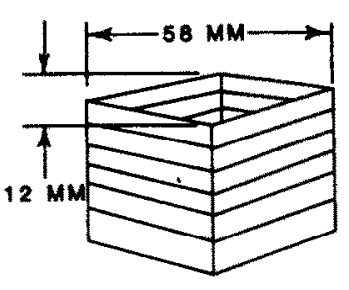

LARGE BOX

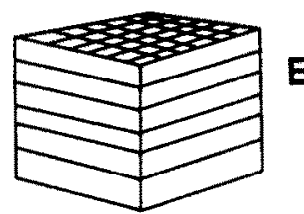

LARGE BOX WITH LID

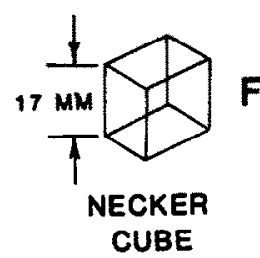

Fig. 1. Line drawings showing the six illustrations with which the subjects were tested. In illustrations (A)-(E), the two fixation points ("front" and "back" corners) were always separated by $12 \mathrm{~mm}$ vertically and about $2 \mathrm{~mm}$ horizontally.

corner was apparently an exterior or an interior corner of the cube. One measurement of vergence state was made for each fixation, in an arbitrarily pre-selected video field about $1 \mathrm{sec}$ before the next ensuing refixation. Slow time trends in estimates of vergence state were commonly evident over a sequence of fixations; these were primarily due to a tendency for the average position of the obstructed eye, during monocular viewing, to drift toward greater divergence (cf. Enright, 1986). Variances of the changes in vergence were therefore estimated from pairwise comparisons of sequential measurements, with $\mathrm{N}$, for calculating standard error of a change in vergence, being half the total number of changes in fixation (i.e. in most cases, $\mathrm{N}=10$ ).

Statistical significance of average singlesubject vergence change with a given target was assessed with a one-tailed t-test (assuming, therefore, that vergence would not, on average, change in the direction opposite to that suggested by target geometry). Consistency across subjects for differences in response between particular target pairs was assessed with twotailed tests on paired intra-subject differences; when two or more estimates, from different test sessions, of the response by a given subject to a given target were available, their average served as the basis for comparison with that person's performance on another target.

Seven of the 9 subjects were females between 20 and 25 years of age; the other two were aged 10 (Subject 7) and 43 years old (Subject 1). All have normal uncorrected acuity in the viewing eye ( $20 / 20$ or better); based on qualitative examination, their saccades appeared normal, as did their pursuit movements in all quadrants. Exoor esophoria, if any, at $3 \mathrm{~m}$ viewing distance was within the normal range $\left(<3^{\circ}\right)$. None of the 9 subjects knew beforehand about "expected" performance in the experiments; and 4 of the 9 had never previously participated in experiments on eye movements.

\section{RESULTS}

\section{Perspective drawings as stimuli}

An excerpt from a recording, made while Subject 1 repeatedly shifted monocular fixation between the upper front and back corners of the drawing of the "small box", is presented in 


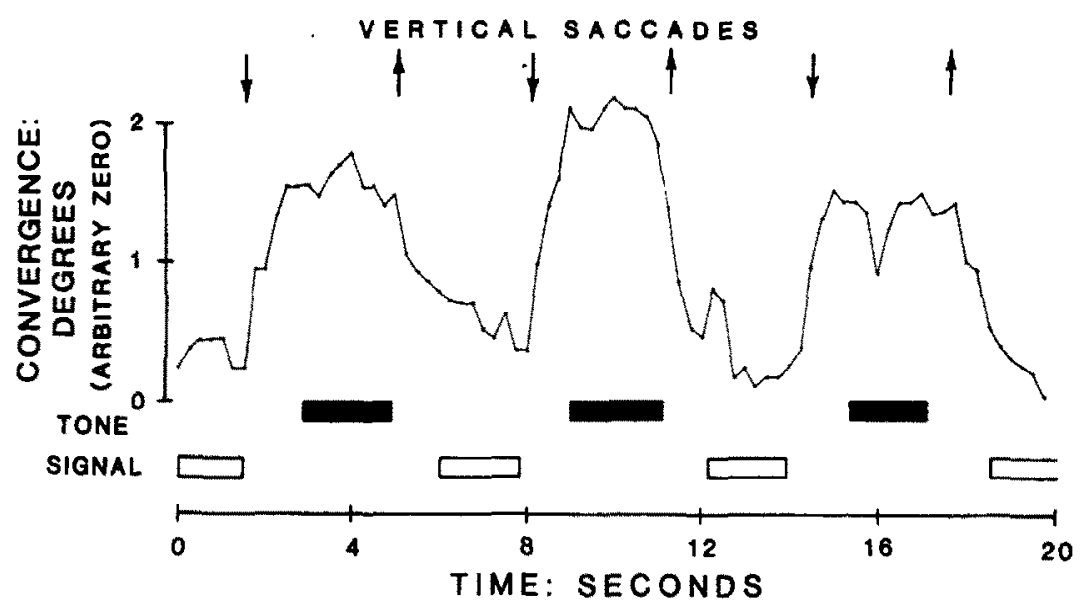

Fig. 2. Vergence measurements during a 20-sec blink-free portion of a recording from Subject 1, during monocular viewing. while she repeatedly changed fixation between the front and back corners in the drawing, "small box"; for the purposes of this figure, every 15 th video frame was measured (4 values/sec). The bars beneath the vergence record show the timing of tone signals, given to indicate which corner was being fixated; dark bars correspond to the tone which indicated the near corner, and lower open bars indicate the tone used for the far corner. Arrows at the top of the diagram indicate timing of the vertical saccades which accompanied the changes in fixation.

Fig. 2. It is clearly evident in this record that the subject converged her eyes when fixating the "near" corner, and diverged for fixation on the "far" corner. Although much of the total vergence change usually occurred in conjunction with the initial change in fixation ("vertical saccades"), there was often also a supplementary drift over the ensuing seconds. Essentially all this post-saccadic eye movement represented additional vergence change by the occluded eye. For a summary evaluation of the entire 21-fixation record, one measurement was taken of vergence state, near the end of each fixation; and differences between sequential values were used to estimate average overall change in vergence, as well as its standard error. Column 2 of Table 1 includes this average value ( 68 arcmin), as well as corresponding values from 5 other test sessions with this subject (1), each on a different day; also presented are comparable estimates for 8 additional subjects. Twenty-one of the 24 tabulated average values are statistically significant at the 0.01 level; and 2 more are significant at the 0.05 level. In brief, all tested subjects showed consistent convergence and divergence, as they changed fixation from the "near" to the "far" corner in the illustration; and the direction of that vergence change would have been appropriate, had they been looking binocularly at a real three-dimensional object like the illustrated box. As can be seen in Table 1 , there was good reproducibility for a given subject, between test sessions; and there were consistent differences between subjects in the average magnitude of the response, with Subjects 1 and 2 showing considerably larger vergence changes than any of the others.

The magnitude of vergence change which would be appropriate for proper binocular fixation on a real box of these dimensions, seen at this viewing distance, can be calculated as follows. The two upper lateral corners of the illustrated box were $29 \mathrm{~mm}$ apart (left-to-right diagonal), and the back corner was about $12 \mathrm{~mm}$ above the front, implying a line-of-sight distance of about $26.4 \mathrm{~mm}$ between front and back corners (SQRT $\left.\left[29^{2}-12^{2}\right]\right)$. The drawing was about $300 \mathrm{~mm}$ from the subject's eyes; and a typical interpupillary spacing is about $60 \mathrm{~mm}$. The geometrically expected vergence response to a real box of these dimensions at this distance is about $1^{\circ}$, based on the equation,

$$
\begin{aligned}
V_{1}-V_{2}=\tan ^{-1} & {[60 /(300-26.4 / 2)] } \\
& -\tan ^{-1}[60 /(300+26.4 / 2)] .
\end{aligned}
$$

(Misperception of the distance to the illustration could, in principle, alter this expectation, but all subjects were aware of the approximate distance to the drawing, which was in place before they entered the bite board.) The observed values of vergence change for Subjects 1 and 2 in response to this target agree reasonably well with this geometrically calculated "expectation", but 
Table 1. Vergence responses to the targets, "small box" and "large box" (near fixation minus far fixation: arcmin \pm SE) $\dagger$

\begin{tabular}{|c|c|c|c|}
\hline \multirow[b]{2}{*}{ Subject } & \multicolumn{2}{|c|}{ Target } & \multirow{2}{*}{$\begin{array}{l}\text { Average difference } \\
\text { (small - large) }\end{array}$} \\
\hline & Small box & Large box & \\
\hline 1 & $\begin{array}{l}65 \pm 8^{* *} \\
68 \pm 8^{* *} \\
72 \pm 7^{* *} \\
73 \pm 7^{* *} \\
82 \pm 7^{* *} \\
87 \pm 5^{* *}\end{array}$ & $\begin{array}{l}56 \pm 9^{* * *} \\
61 \pm 9^{* *} \\
63 \pm 9^{* *} \\
71 \pm 6^{* *}\end{array}$ & +12 \\
\hline 2 & $\begin{array}{l}64 \pm 11^{* *} \\
66 \pm 7^{* *} \\
79 \pm 17^{* *}\end{array}$ & $\begin{array}{l}44 \pm 8^{* *} \\
53 \pm 13^{* *} \\
65 \pm 11^{* *}\end{array}$ & +16 \\
\hline 3 & $\begin{array}{l}28 \pm 10^{* *} \\
34 \pm 7^{* *}\end{array}$ & $\begin{array}{l}31 \pm 4^{* *} \\
39 \pm 6^{* *}\end{array}$ & -4 \\
\hline 4 & $\begin{array}{l}22 \pm 5^{* * *} \\
23 \pm 4^{* *}\end{array}$ & $\begin{array}{l}9 \pm 8 \mathrm{NS} \\
19 \pm 6^{* *}\end{array}$ & +9 \\
\hline 5 & $\begin{array}{l}19 \pm 4^{* *} \\
20 \pm 5^{* *}\end{array}$ & $\begin{array}{l}4 \pm 7 N S \\
15 \pm 6^{*}\end{array}$ & +10 \\
\hline 6 & $\begin{array}{l}16 \pm 6^{* *} \\
23 \pm 6^{* *}\end{array}$ & $\begin{array}{l}10 \pm 6 \mathrm{NS} \\
27 \pm 5^{* *}\end{array}$ & +1 \\
\hline 7 & $\begin{array}{c}6 \pm 9 \mathrm{NS} \\
24 \pm 7^{* *} \\
25 \pm 6^{* *}\end{array}$ & $\begin{array}{l}-2 \pm 5 N S \\
15 \pm 8^{*}\end{array}$ & +12 \\
\hline 8 & $\begin{array}{l}16 \pm 7^{*} \\
20 \pm 6^{* *}\end{array}$ & $\begin{array}{l}12 \pm 7 \mathrm{NS} \\
16 \pm 7^{*}\end{array}$ & +4 \\
\hline 9 & $\begin{array}{l}17 \pm 7^{*} \\
18 \pm 4^{* *}\end{array}$ & $\begin{array}{c}6 \pm 6 \mathrm{NS} \\
24 \pm 6^{* *}\end{array}$ & +2 \\
\hline
\end{tabular}

Average of values in column $4=+7$; median $=+9 . t$-test of average: $P<0.02\left(H_{0}: \quad X=0\right)$; Wilcoxon test of median: $P<0.05\left(H_{0}: \mathrm{Md}=0\right)$.

fIn this and the subsequent tables, "vergence response" was calculated as the vergence state during monocular fixation on the "nearer" corner minus the vergence during fixation on the "far" corner. Positive values in columns 2 and 3 are thus an "appropriate" response; positive values in column 4, however, are anomalous in terms of the relative distances implied by the two drawings.

- $P<0.05$ that true average $\leqslant 0$.

$* P<0.01$ that true average $\leqslant 0$.

NS: Not Significant $(P>0.05)$.

those of the other 7 subjects are appreciably smaller. This tendency toward vergence shortfall is even more evident in the results obtained when the subjects were presented with a line drawing of a box which was shown as being twice as large, but less tilted [Fig. 1(D); data in Table 1, column 3]. Although geometric considerations would lead one to predict responses twice as large, the observed vergence changes were typically less than the responses to the drawing of the smaller box (Table 1, Column 4); and in 6 of the 21 test sessions, the average measured value with the "large box" was not significantly greater than zero.

Partial clarification of this initially unexpected result is provided by tests of two other sorts, conducted with three subjects. In the first of these accessory tests, a drawing identical in proportions to the "small-box" picture, but 10-fold larger, was presented at a 10-fold greater viewing distance $(3 \mathrm{~m})$. The observed vergencechange responses were typically smaller than those of the same subject for the usual small-box illustration at $30 \mathrm{~cm}$, but not 10 -fold smaller, as would have been "appropriate". Subject 1 changed vergence by 60 arcmin \pm 6 arcmin (first test session), and by 41 arcmin \pm 5 arcmin (second session); Subject 2's values had an average of 32 arcmin $( \pm 10)$, and Subject 3's values averaged 16 arcmin $( \pm 5)$. These vergence changes are larger, by a factor of 3-10, than what would have been appropriate for binocular viewing of a box of $29 \mathrm{~cm}$ diagonal, at a distance of $3 \mathrm{~m}$ (expected being 5-6 arcmin). Hence, although the vergence responses to drawings of these sorts are appropriate in direction for the depth relationships implied within the drawing, the size of the vergence changes does not incorporate an appropriate readjustment for variation in the absolute distance to the picture.

A drawing like the "small box", with 2-fold enlargement in all dimensions, was also presented to these three subjects; it was placed at the usual viewing distance of about $30 \mathrm{~cm}$. In terms of angular subtent, that drawing is, of course, an illustration of an object similar in implied size to the "large-box" drawing of Fig. 1(D), but with its opening inclined more steeply: at the same tilt as the "small-box" drawing. The observed vergence changes in response to this "magnified small-box" target were appreciably larger than any of the average responses, by the same subject, to the usual "small-box" illustration. Subject 1 changed vergence by 112 $\operatorname{arcmin} \pm 10$ arcmin (first test session), and 118 arcmin \pm 10 arcmin (second session); Subject 2: 113 arcmin ( \pm 26$)$; and Subject 3: 44 arcmin $( \pm 7)$. These results indicate: (1) that the responses obtained with the usual "small-box" illustration (Column 2 of Table 1) can be considerably enhanced when the target is a larger-scale drawing which is otherwise identical (although scaling of responses, for all three subjects, falls short of simple proportionality); and (2) that the difference in responses between the "small-box" and the other "large-box" illustrations [Fig. 1(A) and 1(D); see Column 4 of Table 1] probably is a consequence of some aspect of the geometric relationships within the figure, which are associated with reducing the apparent tilt of the aperture of the box; the differences in Table 1 do not indicate that a larger illustration-a box shown with the images of its outer edges farther from the 
Table 2. Vergence changes with variants on the "small-box" theme (arcmin \pm SE)

\begin{tabular}{cccccc}
\hline Subject & $\begin{array}{c}\text { A } \\
\text { Small Box } \dagger\end{array}$ & $\begin{array}{c}\text { B } \\
\text { Inverted } \\
\text { Small Box }\end{array}$ & $\begin{array}{c}\text { Difference } \\
(\mathrm{A}-\mathrm{B})\end{array}$ & $\begin{array}{c}\text { Small Box } \\
\text { with Lid }\end{array}$ & $\begin{array}{c}\text { Difference } \\
(\mathrm{A}-\mathrm{C})\end{array}$ \\
\hline 1 & 75 & $42 \pm 8^{* *}$ & +33 & $73 \pm 8^{* *}$ & +2 \\
2 & 70 & $4 \pm 15 \mathrm{NS}$ & +66 & $37 \pm 12^{* *}$ & +32 \\
3 & 31 & $16 \pm 8^{*}$ & +15 & $22 \pm 6^{* *}$ & +9 \\
4 & 23 & $7 \pm 4 \mathrm{NS}$ & +16 & $19 \pm 5^{* *}$ & +4 \\
5 & 19 & $8 \pm 6 \mathrm{NS}$ & +11 & $12 \pm 4^{* *}$ & +8 \\
6 & 20 & $10 \pm 4^{*}$ & +10 & $14 \pm 7^{*}$ & +6 \\
7 & 18 & $18 \pm 5^{* *}$ & 0 & $-1 \pm 6 \mathrm{NS}$ & +19 \\
8 & 18 & $13 \pm 7^{*}$ & +5 & $15 \pm 4^{* *}$ & +3 \\
9 & 17 & $27 \pm 6^{* *}$ & -10 & $25 \pm 5^{* *}$ & -8 \\
\hline
\end{tabular}

Average of values in column $4=+16$; median $=+11$. $t$-test of average: $P=0.06$ $\left(H_{0}: X=0\right)$; Wilcoxon test of median: $P<0.05\left(H_{0}: \mathrm{Md}=0\right)$. Average of values in column $6=+8$; median $=+6$. $t$-test of average: $P=0.06$ $\left(H_{0}: \bar{X}=0\right)$; Wilcoxon test of median: $P=0.05\left(H_{0}: \mathrm{Md}=0\right)$.

tAverages from column 2 of Table 1.

$* P<0.05$ that true average $\leqslant 0$.

$* * P<0.01$ that true average $\leqslant 0$.

NS: Not Significant $(P>0.05)$.

fovea-necessarily produces a reduced vergence-change response, relative to that for a smaller illustration.

A simple (and relatively uninteresting) explanation which might account for a majority of the results described so far is the hypothesis that in a monocular viewing situation, raising the eyes necessarily produces divergence. [Such a phenomenon was reported by Holland (1958), although the effect he measured-an average of about 2-3 arcmin divergence per degree of uptilt-would be far too small to account for the results described here.] A simple test of this hypothesis, in the present context, is to offer the subjects the drawing of the "small box" in an inverted orientation [Fig. 1(B)]; if eye elevation is critical, the upper fixation point-now representing the nearer corner of the opening in the box-should be associated with greater divergence. Results from these tests are presented in Column 3 of Table 2; all the average vergence-change responses are in the direction appropriate for the perspective implication of the drawing (greater convergence for the "nearer" corner), and all are therefore opposite in sign from prediction based on the "angle-ofgaze" hypothesis. Nevertheless, a consistent trend toward reduction in response magnitude is evident in the data; 3 of the 9 average values with the "inverted-box" drawing were not significantly greater than zero (Table 2 , Column 3 ), and 8 of the 9 intra-subject comparisons indicate lesser average vergence change for the upside-down box (Table 2, Column 4). Thus, the "inverted-box" illustration seems to be a somewhat less effective stimulus for evoking vergence changes than the same box shown in an upright orientation.

One of the important ways in which these illustrations imply distance between the fixation points on the corners of the boxes is that the interior siding of the box is partially exposed by the aperture and partially occluded by the front surfaces of the box [Fig. 1(A, B, D)]. Another way of indicating that distance is shown in Fig. 1(C, E); the cross-hatched lids illustrated there fully occlude the inside of the box; instead, the lids provide texture and involve very different sorts of geometric relationships of the line segments between the fixation points. This change, when superimposed upon the "small-box" illustration, resulted in smaller average vergencechange responses for 8 of the 9 subjects (Table 2, column 6); superimposed upon the "large-

Table 3. Vergence changes with variant on the "large-box" theme (arcmin $\pm \mathrm{SE}$ )

\begin{tabular}{cccc}
\hline Subject & $\begin{array}{c}\text { D } \\
\text { Large Boxt }\end{array}$ & $\begin{array}{c}\text { Large Box } \\
\text { with Lid }\end{array}$ & $\begin{array}{c}\text { Difference } \\
\text { (D - E) }\end{array}$ \\
\hline 1 & 63 & $76 \pm 9 * *$ & -13 \\
2 & 54 & $78 \pm 13^{* *}$ & -14 \\
3 & 35 & $38 \pm 5^{* *}$ & -3 \\
4 & 14 & $33 \pm 5^{* *}$ & -9 \\
5 & 9 & $39 \pm 6^{* *}$ & -30 \\
6 & 19 & $23 \pm 5^{* *}$ & -4 \\
7 & 7 & $17 \pm 7^{*}$ & -10 \\
8 & 14 & $17 \pm 5^{* *}$ & -3 \\
9 & 15 & $37 \pm 6^{* *}$ & -22 \\
\hline
\end{tabular}

Average of values in column $4=-13$; median -13. $t$-test of average: $P<0.01 \quad\left(H_{0}: X=0\right)$; Wilcoxon test of median: $P<0.01\left(H_{0}: M d=0\right)$. $* P<0.05$ that true average $\leqslant 0$.

$* P<0.01$ that true average $\leqslant 0$.

tAverage values from column 3 of Table 1 . 


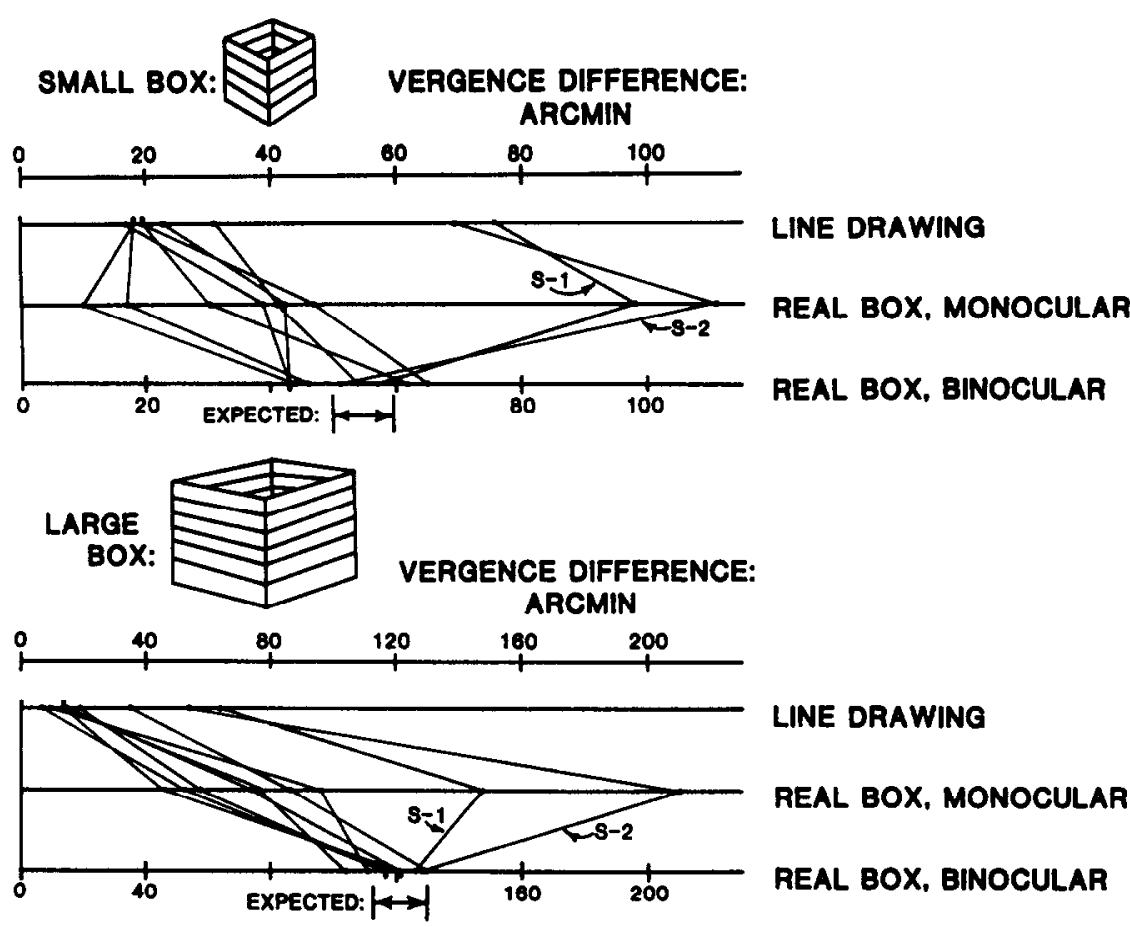

Fig. 3. Average vergence changes made by nine subjects, in changing fixation between the upper front and upper back corners of illustrations and models of rectangular boxes. Upper 3 rows of data are for the drawing of the "small box", and for monocular and binocular viewing of a cardboard box of similar size and appearances; the 3 lower rows of data are equivalent values for the drawing of the "large box", and for its corresponding cardboard model. "Expected" values apply to "real-box-binocular" data only; they represent the inter-subject range of vergence changes predicted on the basis of target geometry, assuming perfect binocular fixation, precise calibration, etc. Points which are connected by diagonal lines represent same-subject estimates, with $S_{1}$ and $S_{2}$ being the data for Subjects 1 and 2 respectively.

box" illustration, the cross-hatched lid enhanced the average response of all 9 subjects (Table 3). This contrast in results indicates that while the magnitude of vergence responses can be consistently altered by changes in components of a drawing, the nature of that effect depends upon more than simply the qualitative features of content, such as open-box vs boxwith-lid.

\section{Real objects as stimuli}

In order to compare the magnitude of these responses, evoked by perspective stimuli, to the vergence-change responses which occur during viewing of real objects, three-dimensional models were constructed, marked with black lines to resemble the drawings, and presented to the subjects with an orientation such that the monocular impression approximated that of the "small-box" or the "large-box" illustrations [Fig. 1(A or D)]. The vergence-change responses to these "real boxes", during both monocular and binocular viewing, are summarized in Table 4; and Fig. 3 contains a graphical summary of the average values, together with the vergencechange responses to the corresponding perspective drawings. The relationships shown in Fig. 3 indicate that the responses to perspective stimuli in the drawing were an appreciable fraction of the total binocular response to the small cardboard box; and a lesser but non-negligible fraction of the binocular response to the large box. That conclusion should not, however, be overgeneralized; it cannot be directly applied to drawings and objects presented at other viewing distances (see Discussion).

The "expected" values for binocular viewing shown in Table 4 (Columns 4 and 7) were derived from geometric calculations, based on measurements, to the nearest $\mathrm{mm}$, of target distance and inter-ocular spacing. Averaged over all subjects, the observed vergence changes during binocular viewing were slightly smaller than expected (a difference of 3 arcmin for the smaller box and 5 arcmin for the larger). Several of the single-subject data sets, however, involve larger and statistically significant departures from expectation; the most likely source of 
Table 4. Vergence changes with real boxes (arcmin $\pm \mathrm{SE}) \dagger$

\begin{tabular}{ccccccc}
\hline Subject & $\begin{array}{c}\text { Monocular } \\
\text { viewing }\end{array}$ & $\begin{array}{c}\text { Small box } \\
\text { Binocular } \\
\text { viewing }\end{array}$ & $\begin{array}{c}\text { Expected } \\
\text { (binocular) }\end{array}$ & $\begin{array}{c}\text { Monocular } \\
\text { viewing }\end{array}$ & $\begin{array}{c}\text { Large box } \\
\text { Binocular } \\
\text { viewing }\end{array}$ & $\begin{array}{c}\text { Expected } \\
\text { (binocular) }\end{array}$ \\
\hline 1 & $98 \pm 9$ & $57 \pm 4$ & 57 & $149 \pm 8$ & $126 \pm 4$ & 123 \\
2 & $111 \pm 12$ & $51 \pm 7$ & 54 & $210 \pm 29$ & $128 \pm 4^{*}$ & 119 \\
3 & $41 \pm 5$ & $54 \pm 6$ & 50 & $86 \pm 6$ & $129 \pm 5^{* *}$ & 113 \\
4 & $42 \pm 7$ & $43 \pm 7^{*}$ & 58 & $76 \pm 6$ & $104 \pm 5^{* *}$ & 125 \\
5 & $47 \pm 6$ & $65 \pm 6$ & 56 & $96 \pm 4$ & $111 \pm 5$ & 121 \\
6 & $30 \pm 7$ & $62 \pm 7$ & 57 & $57 \pm 11$ & $117 \pm 7^{*}$ & 133 \\
7 & $10 \pm 7 \mathrm{NS}$ & $44 \pm 3^{*}$ & 50 & $52 \pm 5$ & $117 \pm 4^{*}$ & 125 \\
8 & $16 \pm 5$ & $46 \pm 3^{* *}$ & 59 & $45 \pm 6$ & $121 \pm 7$ & 127 \\
9 & $39 \pm 8$ & $43 \pm 7$ & 54 & $74 \pm 7$ & $121 \pm 7$ & 130 \\
Average & 48 & 52 & 55 & 88 & 119 & 124 \\
Median & 41 & 51 & 56 & 76 & 121 & 125 \\
\hline
\end{tabular}

†All values in columns 2,3,5 and 6 are significantly greater than zero at the 0.01 level, except for Subject 7 in column 2, labelled NS.

"Observed value during binocular viewing differs from "Expected" at the 0.05 level; see, however, Discussion for the interpretation of this significance.

**observed value during binocular viewing differs from "Expected" at the 0.01 level; see, however, Discussion for the interpretation of this significance.

those deviations is faulty fixation, either during calibration measurements or during testing with the boxes used as targets (see Discussion).

\section{Changes in pupil size}

The responses of the subjects to the cardboard boxes, during both monocular and binocular viewing, differed in an important qualitative way from the responses to the perspective drawings: when fixation was changed back and forth while viewing the model, there were usu- ally consistent changes in pupil size: an expected component of the "near reflex" (near fixation associated with pupil constriction: Lowenstein and Lowenfeld, 1962); but when vergence changes occurred in response to the perspective drawing, appropriately correlated changes in pupil size were decidedly not typical (Table 5). It is evident (Fig. 3) that the average vergencechange responses to the drawings were typically somewhat less than the monocular responses to the real boxes; assuming that there should be a

Table 5. Changes in pupil diameter with changes in fixation distance (micrometers $\pm S E$ ) $\dagger$

\begin{tabular}{cccc}
\hline Subject & $\begin{array}{c}\text { Large real box } \\
\text { (binocular viewing) }\end{array}$ & $\begin{array}{c}\text { Small real box } \\
\text { (monocular viewing) }\end{array}$ & $\begin{array}{c}\text { Drawing of small box } \\
\text { (monocular viewing) }\end{array}$ \\
\hline 1 & $139 \pm 29^{* *}$ & $63 \pm 18^{* *}$ & $-49 \pm 23$ (NS) \\
2 & $179 \pm 23^{* *}$ & $75 \pm 23^{* *}$ & $+5 \pm 18 \mathrm{NS}$ \\
3 & $292 \pm 32^{* *}$ & $158 \pm 32^{* *}$ & $+34 \pm 20 \mathrm{NS}$ \\
4 & $179 \pm 43^{* *}$ & $136 \pm 55^{*}$ & $-27 \pm 52 \mathrm{NS}$ \\
5 & $216 \pm 30^{* *}$ & $126 \pm 44^{* *}$ & $-31 \pm 50 \mathrm{NS}$ \\
6 & $207 \pm 29^{* *}$ & $-10 \pm 24 \mathrm{NS}$ & $-94 \pm 38(\mathrm{NS})$ \\
7 & $149 \pm 49^{* *}$ & $47 \pm 37 \mathrm{NS}$ & $-44 \pm 41 \mathrm{NS}$ \\
8 & $193 \pm 32^{* *}$ & $84 \pm 45 \mathrm{NS}$ & $+1 \pm 41 \mathrm{NS}$ \\
9 & $151 \pm 36^{* *}$ & $90 \pm 44^{*}$ & $-61 \pm 31$ (NS) \\
Average & $189 \pm 16$ & $85 \pm 17$ & $-30 \pm 13$ \\
Median & 179 & 84 & -31 \\
Vergence change: & $119 \pm 3$ arcmin & $48 \pm 11$ arcmin & $34 \pm 8$ arcmin \\
& (see Table 4 for details) & (see Table 1) \\
Ratio of averages: & & 1.77 & -0.88 \\
Pupil/vergence & 1.59 & &
\end{tabular}

FPositive values represent smaller pupil when the fixated corner was nearer, based on the usual linkage between convergence and pupillary constriction.

†This is the average vergence change for the 9 test sessions, 1 per subject, in which pupil size was also measured.

$* P<0.05$ that true average $\leqslant 0$.

$* * P<0.01$ that true average $\leqslant 0$.

NS: Not Significant $(P>0.05)$.

(NS): Value would have been significantly different from zero, in the negative direction, at the 0.05 level, had a two-tailed test been used. 
positive relationship between the magnitudes of vergence change and of change in pupil size, the pupillary responses to the drawing should be expected to be somewhat smaller than those to the models. On average, however, some sort of positive response ought to have been expected, and in fact there was, instead, a tendency in the opposite direction; the negative values, for 6 subjects out of 9 (Table 5, Column 4) indicate greater pupil dilation in conjunction with convergence and fixation on the "near" corner of the illustration. The implications of this unexpected result will be considered below (Discussion).

\section{The Necker cube as stimulus}

The vergence changes described up to this point have involved a concurrent vertical shift in the direction of gaze: the eyes had to move upward or downward by about $2.5^{\circ}$. Qualitatively similar vergence changes in response to a line drawing can also, however, be evoked by an illustration of a Necker cube [Fig. 1(F)], while the viewing eye maintains fixation on a single point within the drawing. In these tests, the subjects were instructed to give the appropriate tone signal only when they both perceived the fixated corner as being, say, the frontal exterior corner, and also had the impression that they could "hold" the perception for at least 2-3 sec; then, after several sec of a given perception and tone signal, they were instructed to "invert" the perception, and give the corresponding, alternative tone only after they had the impression that this opposite perception (fixated corner is interior) could be "held". This instruction sometimes led to a lag of as much as 8 to $10 \mathrm{sec}$ between signals, and between measurements. (Such instructions eliminate the possible ambiguity in relating vergence state to perception, which might be introduced by the reaction time for pressing or releasing the switch, during less stable perception.)

Results from these tests are summarized in Table 6. For 2 of the subjects, the vergencechange response to the Necker cube was not significantly greater than zero (Table 6, Column 2), and for 7 of the 9 subjects, the responses were somewhat smaller than the average value with the "small-box" illustration. In general, there was agreement in direction of the vergence responses with observations from the unambiguous perspective drawings, but there was also a conspicuous qualitative difference between results from these two kinds of stimuli.
With the Necker cube, the subjects-including those 2 whose vergence responses were smallest-showed conspicuous changes in pupil diameter, in the direction expected for the "near reflex" (constriction when the fixation point was perceived as being the frontal, exterior corner: Table 6, Column 3). No such pupillary response occurred with the perspective drawing of the small box (Table 5, Column 4).

In view of the relatively small size of the vergence changes obtained with the Neckercube picture, the observed pupil changes shown in Table 6 are in fact remarkably large: the average ratio of pupil change to vergence change (5.33) was appreciably greater than the value measured during monocular or binocular viewing of a real cardboard box (1.59 and 1.77, respectively); the latter values presumably express the usual "near reflex". As another indication of this phenomenon, note that the vergence change in response to the Necker cube was smaller than that in response to the monocular view of the small cardboard box for 8 subjects of 9; but the changes in pupil diameter for the Necker cube were larger than those for the monocular view of the small box for 7 subjects of 9. In any case, the contrast between these pupil responses to the Necker cube and those obtained with the drawing of the "small box" is striking, suggesting that the two results may represent fundamentally different phenomena.

\section{DISCUSSION}

\section{Reliability of the measurements}

How reliably do the data presented here

Table 6. Changes in vergence and in pupil diameter: responses to Necker cube

\begin{tabular}{ccc}
\hline Subject & $\begin{array}{c}\text { Vergence changes } \\
(\text { arcmin } \pm \mathrm{SE}) \dagger\end{array}$ & $\begin{array}{c}\text { Changes in pupil diameter } \\
\text { (Micrometers } \pm \mathrm{SE}) \dagger\end{array}$ \\
\hline 1 & $78 \pm 8^{* *}$ & $69 \pm 19^{* *}$ \\
2 & $41 \pm 10^{* *}$ & $52 \pm 24^{*}$ \\
3 & $26 \pm 6^{* *}$ & $92 \pm 41^{*}$ \\
4 & $20 \pm 10^{*}$ & $220 \pm 14^{* *}$ \\
5 & $16 \pm 8^{*}$ & $210 \pm 63^{* *}$ \\
6 & $24 \pm 8^{* *}$ & $135 \pm 40^{* *}$ \\
7 & $40 \pm 7^{* *}$ & $96 \pm 53 \mathrm{NS}$ \\
8 & $3 \pm 6 \mathrm{NS}$ & $382 \pm 106^{* *}$ \\
9 & $7 \pm 6 \mathrm{NS}$ & $104 \pm 35^{* *}$ \\
Average & $28 \pm 8$ & $151 \pm 35$ \\
Median & 24 & 104 \\
\hline
\end{tabular}

Ratio of averages: pupil/vergence $=5.33$.

†Positive values for vergence change and for change in pupil diameter represent greater convergence and smaller pupil, respectively, when the fixated comer was perceived as being nearer.

$* P<0.05$ that true average $\leqslant 0$.

$* * P<0.01$ that true average $\leqslant 0$.

NS: Not Significant $(P>0.05)$. 
describe true vergence movements? Reproducibility of the measurements, which is one component of reliability, is adequately indicated by standard errors, and the similarity of results, for a given subject and target, in different test sessions. Those values, however, do not address the possibility of bias in the measurement technique; and when vergence state is estimated on the basis of single-view monitoring of the position of each eye, serious systematic errors can arise due to lateral translation of the globe. Consistent ocular translation of that sort has been documented for large vergence changes (on the order of $20^{\circ}$ ) associated with either fusional stimuli or accommodation stimuli (Enright, 1984a). Measurements with the present monitoring system indicate that this sort of problem is of quite small magnitude when the vergence changes are small and when results are averaged over an appreciable number of replicate trials. For a previous publication (Enright, 1986), recordings from 5 subjects were measured, each for 4 target locations (vergence changes of $2-3^{\circ}$ ), with 12 tests per target. When these 20 estimates of average steady-state vergence change, during binocular viewing, were compared with expectations from target geometry, the observed values averaged about 4 arcmin less than predicted (Enright, 1986).

Similarly good agreement with geometric expectation, in overall average, is evident in Table 4 here; the mean observed value during binocular viewing of the small cardboard box was about 3 arcmin less than predicted, and with the larger box, the average deviation was about 5 arcmin in the same direction. Nevertheless, several of the single-subject values differ from expectation to a statistically significant extent. That statistical significance must be tempered by the recognition that it assumes perfect calibration measurements; and also assumes that the "expected" values are known without error. There is, of course, some residual uncertainty associated with the calibrations, as well as in measurement of interocular spacing and target distance, and in the assumed locations of the center of ocular rotation and of the nodal point (assumed values of $14 \mathrm{~mm}$ and $8 \mathrm{~mm}$ behind the upper eyelid, respectively). Even making allowance for these possible sources of error, however, some of the single-subject deviations are sufficiently large-note, particularly, those of Subject 4, which, for both targets, depart most extremely from predictions-as to suggest that either faulty fixation or systematic ocular trans- lation sometimes contributed to the data. Despite those problems, the agreement between observed and expected values shown in Table 4 is sufficiently good to lend credibility to the other data, obtained during monocular viewing, when no such comparison is possible.

\section{Relationship with proximal vergence}

If one accepts, on this basis, that vergencechange responses to perspective drawings are a real and reproducible phenomenon, the next issue of importance is where this sort of result fits into existing knowledge about the oculomotor system. There is apparently no precedent in the literature for testing of perspective drawings as stimuli for vergence change, but a somewhat related category of response, now called "proximal vergence", has long been known. Maddox (1893), in the first definitive classification of disjunctive eye movements, proposed that "knowledge of nearness" has an effect on vergence state; and responses of this general sort have subsequently been described under many different names, including psychological vergence, instrument vergence, perceptual vergence and psychic vergence (Alpern, 1962). Of particular interest here are several publications (e.g. Ittelson and Ames, 1950; Hofstetter, 1950; Alpern, 1958) which have dealt with an effect of target size upon steadystate vergence, based on the interpretation that an anomalously large or small target-say a much enlarged picture of a playing card-will lead to the impression that the seen object is closer than it really is; and that this misperceived distance will evoke a vergence response in the corresponding direction. More recently, Collewijn et al. (1986; see, also, Erkelens and Regan, 1986) have described a dynamic effect in which cyclic changes in target size produced cyclic vergence movements, with peak-to-peak oscillations in response of about 10 arcmin; in contrast with earlier reports (e.g. Alpern, 1958), they found no sustained response to target size. The most common situation in which "proximal vergence" has been measured, however, is in the clinical study of accommodation vergence; quite often, the actual target distance, known by the subject, has been found to have a small but detectable effect on phoria, when the accommodative stimulus is held constant (Hokoda and Ciuffreda, 1983).

Although the vergence changes described here, in response to perspective drawings, involve a somewhat different stimulus than that in 
the other phenomena currently embraced by the term "proximal vergence", the similarities are sufficiently great that it seems appropriate-at least provisionally - to consider perspective vergence as a subcategory of proximal vergence. The basis for residual uncertainty lies in the finding that vergence changes associated with perspective stimuli were not accompanied by corresponding changes in pupil diameter (Table 5). These responses were not, then, a typical expression of activation of the "near reflex", since at least one component of "neartriad" responses (Semmlow and Kung, 1983) was absent. That anomaly suggests the possibility that perspective vergence may involve fundamentally different pathways to the oculomotor nuclei.

\section{"Paradoxical monocular stereopsis"}

Although actual measurements of vergence responses to perspective illustrations have apparently not been previously reported, Claparède (1904) speculated that during monocular inspection of a photograph or picture, convergence-divergence changes of this sort might arise, responses which would be overridden by fusion, during binocular viewing. The primary purpose of Claparède's brief note (1904) was to call attention to a remarkable and neglected sort of depth perception, which he called "paradoxical monocular stereopsis", and which can often be obtained by monocular inspection of a photograph or picture. It was his notion that the posulated oculomotor response might be responsible for this monocular depth impression, as well as for the fact that it is immediately abolished when the other eye is opened. (The term, "stereopsis", is usually defined as a binocular phenomenon. Claparède's anomalous use of that word for a monocular perception was apparently intended to emphasize that the sense of depth can be fully as compelling as a view through a stereoscope; as Pirenne (1970) commented, the subjective impression is somehow like stereopsis.) This stereopsis-like monocular depth perception has been redescribed in a small number of other publications (Streiff, 1923; Ames, 1925; Schlosberg, 1941, Pirenne, 1970), but none of these later authors has endorsed Claparède's hypothesis, that vergence movements might explain the subjective impression. The results here (see, also, Enright, 1987) demonstrate that during monocular viewing of a picture, vergence changes do indeed arise, thereby confirming one aspect of Claparède's speculation; but whether such vergence changes are causally related to this sort of monocular depth perception must remain an open question.

\section{Perspective stimuli during binocular viewing}

While most of the data described here were obtained with one eye occluded, it seems very probable that perspective-like stimuli, as they are provided by a three-dimensional environment, contribute to vergence responses during our ordinary, day-to-day binocular visual performance. The data suggest that perspective vergence is a supplementary or backup system, comparable with accommodation vergence, which can move the eyes in the direction required for fusion. One way of assessing how significant this contribution might be is implied by Fig. 3. In that graph it seems clear that the responses to perspective stimuli can, for most subjects, account for a relatively large fraction of the total binocular response to the small cardboard box; and for an appreciably lesser fraction of binocular responses to the large-box model. There are, however, several good reasons for hesitation, in attempting to generalize about or quantify these relationships.

One of the assumptions which would underlie any such interpretation is that the influences of the different stimuli which can produce vergence change are fully additive; this notion can be traced back at least to the formulations of Maddox (1893), but it has never been rigorously justified (Morgan, 1983). Another of the assumptions is that accommodation stimuli and perspective stimuli, as perceived during monocular viewing, have the same effect during binocular viewing-ignoring the possibility that the previously occluded eye, which now also perceives accommodation and perspective stimuli, might make a supplementary contribution to vergence movement as a consequence of those stimuli. In other words, the difference in response between monocular and binocular viewing may not be due exclusively to disparity stimuli. Another uncertainty involved in any attempt to draw firm conclusions from Fig. 3 is the tacit implication that with a monocular view of the cardboard box, only accommodation stimuli could have produced greater vergence changes than those evoked by the drawing. Accommodation stimuli no doubt contributed to the greater response, but other stimuli, associated with the model and absent in the drawing 
(e.g. texture; distribution of light and shadow) may also have played a role.

Still other difficulties in any attempt to quantify the relative contribution of perspective stimuli under ordinary viewing conditions are suggested by a preliminary experiment conducted in collaboration with Dr Clifton Schor, using an eye-monitoring system which permits constriction of the effective ocular aperture by optical means (the visual equivalent of viewing through a pinhole). The subject was presented with a monocular view of the "small-box" drawing [Fig. 1(A)] at about $40 \mathrm{~cm}$ distance; and, with drug-induced pupil dilation, he alternated fixation between "front" and "back" corners of the illustration. When the artificial pinhole pupil was interposed, "appropriate" vergence changes occurred, and they were of extraordinarily large magnitude ( $\mathrm{ca} 2^{\circ}$ ), compared with the results of Table 1 . When, however, the dilated natural pupil was fully exposed to the target, the subject's steady-state vergence changes were smaller by at least a factor of 4 . The clear implication of this contrast is that the magnitude of the vergence-change response to perspective stimuli depends upon pupil size, and hence can be expected to vary to some extent with ambient illumination. Presumably the effect of pupil size upon the magnitude of perspective vergence arises because a pinhole pupil "opens the loop" of the accommodation feedback system; and greater freedom of accommodation leads to different vergence changes through the known coupling between accommodation state and vergence ("accommodation vergence" and "convergence accommodation"; see Schor, 1983; Schor and Kotulak, 1986; Semmlow and Hung, 1983). Nevertheless, there are several possible interpretations for that result. For example, the extent of a steady-state vergence response to perspective stimuli may be limited by the viewer's tolerance for accommodation-induced image blur. In other words, larger vergence changes, which might, in principle, result from the perspective stimuli provided, would lead to larger changes in accommodation; and the resulting image blur may then inhibit full expression of that vergence response. An alternative interpretation, favored by $\mathrm{Dr}$ Schor and his collaborators (McLin et al., 1987; and personal communication) is that accommodation vergence plays a major role in vergence responses to monocular distance cues; that the primary response to such stimuli may be mediated by the accommodative system, which, then, secondarily, produces the vergence response reported here.

Probably the most serious problem, however, in attempting to generalize on the basis of Fig. 3, is that these results were obtained with targets at $30 \mathrm{~cm}$ viewing distance; and that very different interpretations are probably applicable to similar stimuli from targets at other distances. The importance of this issue is shown by the accessory experiments, in which a 10-fold larger drawing was presented at a 10-fold greater distance. The vergence changes evoked by changes of monocular fixation within this enlarged drawing were typically somewhat smaller than those at $30 \mathrm{~cm}$ viewing distance, but the reduction in response was far less than sufficient to match the 10-fold reduction which would be geometrically "appropriate" for a $29-\mathrm{cm}$ box, during binocular viewing at 3-m distance. Thus the observed vergence changes in response to perspective stimuli were much too large for an equivalent real object. This kind of over-response to perspective stimuli-if it were also occur during binocular viewing of a real box-would require fusional convergence, when changing fixation from a nearer to a more distant fixation point. An inverted fusional response to disparity stimuli is in fact also implied for Subjects 1 and 2 , even at $30 \mathrm{~cm}$ viewing distance, because of their strong response during monocular viewing of the cardboard boxes (note direction of change, from monocular to binocular viewing, for $S_{1}$ and $S_{2}$ in Fig. 3.)

On the basis of the many reservations described above, a quantitative extrapolation of the data in Fig. 3 would be hazardous indeed. Nevertheless, the qualitative conclusion seems justified, that perspective stimuli are an important and previously neglected contributor to vergence changes during ordinary binocular viewing. At near viewing distances, accommodation stimuli may with some targets contribute more strongly to total binocular vergence changes than perspective stimuli (e.g. "large box" in Fig. 3); but at greater viewing distances, it seems likely that perspective stimuli would dominate over accommodation stimuli. In all cases, of course, disparity stimuli remain the final arbiter, in deciding how much vergence change is required for proper binocular congruence.

\section{The Necker cube}

The experiments in which a Necker cube served as the stimulus played a critical role in 
the development of this research. The changes in pupil diameter, which occur in conjunction with the "switchover" in the perceived spatial configuration of the Necker cube (Table 6, Column 3), were noted and measured before any of the other phenomena described here; and the next measurements made were of the associated changes in vergence (Table 6, Column 2). These results are, I think, of considerable interest in their own right: changes in vergence and in pupil size represent a reasonably reliable way of "reading a person's mind", as one of the subjects phrased it. Research on the vergence responses to other, unambiguous targets was thereafter undertaken, and it was only much later that the absence of corresponding pupillary responses with the perspective drawings was recognized and measured (Table 5, Column 4). That latter, critical observation strongly suggests that the vergence-change responses to the Necker cube and those to the perspective drawings are not closely related phenomena. Vergence responses to a Necker-cube may well involve voluntary activation of the normal vergence centers which are associated with the "near triad" (Semmlow and Hung, 1983)-although the pupil-change response were, in fact, unusually large compared with those which accompany accommodation vergence and disparity vergence (compare Table 6 with Columns 2 and 3 of Table 5). The absence of a consistent corresponding pupillary response to perspective stimuli suggests that very different control centers may be involved in perspective vergence. With the Necker cube as target, the steady-state location of retinal stimulation remains unchanged, but there is a perceptual shift in its interpretation; with perspective illustrations, the changes in fixation point produce altered distributions of stimuli on the retina; but whether these factors are critical for the observed differences in overall oculomotor response remains uncertain.

\section{Stimulus properties which evoke perspective ver-} gence

A full exploration of the characteristics of illustrations which can evoke vergence responses will be complicated project, and the results presented here indicate that intuitively plausible first guesses about what components of a drawing are important can be badly mistaken. For example, a modest reduction in the inclination of the aperture of the enlarged box-and the associated rearrangement of the drawing-led to a drastic reduction in the vergence-change responses. Doubling the size of the small-box illustration led to appreciably stronger vergence responses-but not two-fold larger. Superimposing a cross-hatched lid on the "large-box" drawing consistently enhanced the vergencechange responses; but when a similar appearing lid, with finer textured cross-hatching, was superimposed on the "small-box" drawing, it reduced the responses. The cross-subject consistency of these changes in response magnitude demonstrates that we have exposed relatively general properties of the oculomotor-response system, and yet none of these differences in response seems to be readily predictable on any sort of a priori principles.

In the line drawings used as stimuli here, the implication of depth is conveyed by what is called "linear perspective." The full spectrum of techniques available to an artist to convey depth in a perspective illustration, however, includes a variety of other factors as well: such considerations as the distribution of light and shadow; superposition; composition (more distant objects usually above closer ones); gradients in texture, and the like. In experiments to be described elsewhere (Enright, 1987), directionally appropriate vergence-change responses were also obtained with monocular viewing of complex artwork in which linear perspective played a negligible role. Since this kind of response can be evoked by a broad spectrum of stimulus configurations used by artists to imply relative positions of objects in depth, its utility may extend to far more general sorts of viewing context than one might suspect, if attention were to be confined only to situations with conspicuous elements of linear perspective.

Acknowledgements-Research supported by Grants BNS 83-03398 and BNS 85-19616 from the National Science Foundation. Encouragement in this line of research was given by $\mathrm{Dr} \mathrm{H}$. Collewijn, and valuable comments on the manuscript were offered by Dr C. Schor.

\section{REFERENCES}

Alpern M. (1958) Vergence and accommodation. Can change in size induce vergence movements? $A . M . A$. Arch. Ophthal. 60, 355-357.

Alpern M. (1962) Types of movement. In The Eye, Vol. III (Edited by Davson H.), pp. 63-151 (esp. p. 137). Academic Press, New York.

Ames A. Jr (1925) The illusion of depth from single pictures. J. opt. Soc. Am. 10, 137-148.

Claparède E. (1904) Stereoscopie monoculaire paradoxale. Ann. d'Oculist. 132, 465-466.

Collewijn H., Erkelens C. J. and Regan D. (1986) Ocular 
vergence movements induced by changing size and disparity. Invest. Ophthal. visual Sci., Suppl. 27, 80.

Enright J. T. (1984) Changes in vergence mediated by saccades. J. Physiol., Lond. 350, 9-31.

Enright J. T. (1984a) Saccadic anomalies: vergence induces large departures from ball-and-socket behavior. Vision Res. 24, 301-308.

Enright J. T. (1986) Facilitation of vergence changes by saccades: influences of misfocussed images and of disparity stimuli in man. J. Physiol., Lond. 371, 69-87.

Enright J. T. (1987) Art and the oculomotor system: Perspective illustrations evoke vergence changes. To be published.

Erkelens C. and Regan D. (1986) Human ocular vergence movements induced by changing size and disparity. J. Physiol., Lond. 379, 145-160.

Hofstetter H. W. (1950) Accommodation, convergence and their relation to apparent distance: a criticism. J. Psychol. 30, 393-394.

Hokoda S. C. and Ciuffreda K. J. (1983) Theoretical and clinical importance of proximal vergence and accommodation. In Vergence Eye Movements: Basic and Clinical Aspects (Edited by Schor C. M. and Ciuflreda K. J.), pp. 75-97. Butterworths, London.

Holland G. (1958) Untersuchung ueber den Einfluss der Fixationsentfernung und der Blickrichtung auf die horizontale Heterophorie (Exo- und Esophorie). Albrecht $v$. Graefes Arch. Ophthal. 160, 144-160.

Ittelson W. H. and Ames A. Jr (1950) Accommodation, convergence and their relation to apparent distance. J. Psychol. 30, 43-62.
Lowenstein O. and Lowenfeld I. E. (1962) The pupil. In The Eye, Vol. III, (Edited by Davson H.), pp. 231-267. Academic, New York.

McLin L., Schor C. M. and Kruger P. (1987) Volitional and size induced changes in accommodation and vergence. Invest. Ophthal. visual Sci., Suppl. 28, 317.

Maddox E. E. (1893) The Clinical Use of Prisms; and the Decentering of Lenses, 2nd edn. John Wright, Bristol.

Morgan M. W. (1983) The Maddox analysis of vergence. In Vergence Eye Movements: Basic and Clinical Aspects (Edited by Schor C. M. and Ciuffreda K. J.), pp. 15-21. Butterworths, London.

Pirenne M. H. (1970) Optics, Painting and Photography, p. 95. Cambridge Univ. Press.

Schlosberg H. (1941) Stereoscopic depth from single pictures. Am. J. Psychol. 54, 601-605.

Schor C. M. (1983) Fixation disparity and vergence adaptation. In Vergence Eye Movements: Basic and Clinical Aspects (Edited by Schor C. M. and Ciuffreda K. J.), pp. 465-516. Butterworths, London.

Schor C. M. and Kotulak J. (1986) Dynamic interactions between accommodation and vergence are velocity sensitive. Vision Res. 26, 927-942.

Semmlow J. L. and Hung G. K. (1983) The near response: theories of control. In Vergence Eye Movements: Basic and Clinical Aspects (Edited by Schor C. M. and Ciuffreda K. J.), pp. 175-195. Butterworths, London.

Streiff J. (1923) Die binoculare Verflachung von Bildern, ein vielseitig bedeutsames Sehproblem. Klin. Monatsbl. Augenheilk. 70, 1-17. 